\title{
Effect of Steering Limit Constraints on the Performance of Variable Stiffness Laminates
}

\author{
Daniël M. J. Peeters ${ }^{1}$, Mostafa M. Abdalla ${ }^{1}$ \\ ${ }^{1}$ Delft University of Technology, The Netherlands, d.m.j.peeters@tudelft.nl
}

Keywords: Optimisation, variable stiffness, lamination parameters, manufacturability

\begin{abstract}
A method to optimise the fibre angle distribution of variable stiffness laminates is proposed. The proposed method integrates a fibre angle retrieval step with a fibre angle optimisation procedure. A multi-level approximation approach is used in combination with the method of successive approximations. First, fibre angle retrieval is done by approximating the structural responses based on the optimal stiffness distribution found using lamination parameters. The full fibre angle optimisation is done by updating the approximations based on the current stacking sequence. It is shown for a bucking optimisation with a stiffness constraint that the number of finite element analyses reduces significantly by starting the optimisation from the optimal stiffness distribution rather than from a user-specified stacking sequence. Next, it is shown that updating the approximations also leads to considerable improvements over fibre angle retrieval. Similar promising results are obtained for a stress optimisation problem.
\end{abstract}

\section{Introduction}

Today, composite materials are frequently used in the aviation industry and the first composite-dominated planes like the B-787 or A400M are being built. Traditionally, fibres within a layer have the same orientation, leading to constant stiffness properties. As manufacturing technology has evolved, for example the advent of automated fibre placement machines, the fibre orientation of a layer can be varied continuously leading to varying stiffness properties that can be best tailored for the applied loads. These composites are called variable stiffness laminates (VSL).

To exploit the possibilities of VSL fully, a three-step approach has been developed. The first step is to find the optimal stiffness distribution in terms of the lamination parameters. This is discussed in detail in Ijsselmuiden $[1,2]$. The second step is to find the optimal manufacturable fibre angle distribution, the focus of this paper $[3,4,5]$. The third step is to retrieve the fibre paths, discussed in Blom [6]. A schematic overview of this approach is shown in Figure 1.
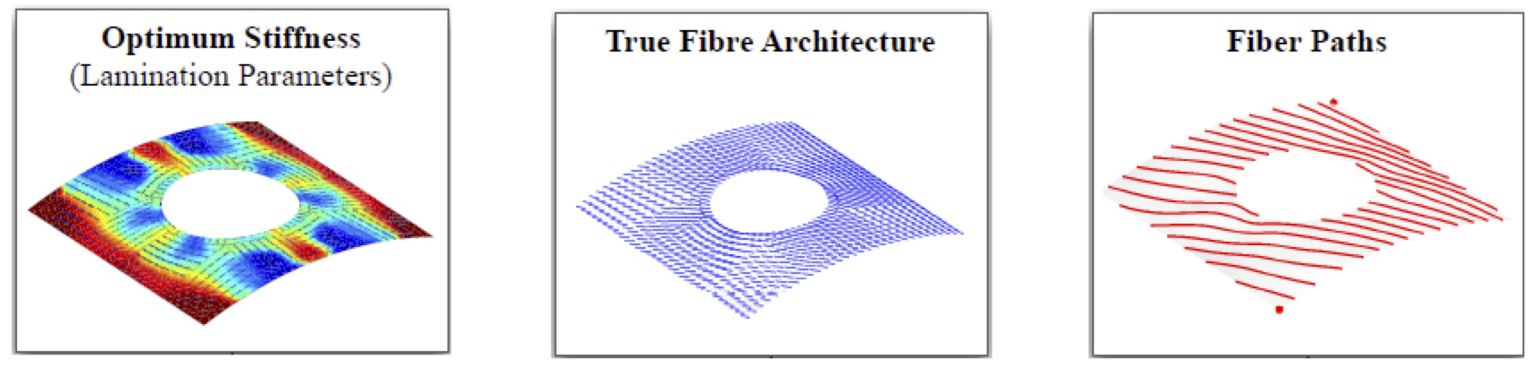

Figure 1. schematic overview of the three-step approach [1]

One of the largest problems in optimising VSL, is taking manufacturability into account. To do this, linearly varying fibre angles are used which has given promising, manufacturable, results [7, 8, 9, 10,11, 12]. The use of linearly varying fibre angle per bay, for stiffened plates, has also been investigated, and again it has been shown that varying the fibre angles leads to better performance $[13,14]$. Direct parametrisation of the tow paths using Lagrangian polynomials, splines or NURBS (Non -Uniform Rational B - Splines) has also been used. Large, manufacturable, improvements in buckling load were found, but the result is dependant of the basis functions you chose to incorporate $[15,16,17]$. Hence, the total potential of VSL has not been exploited due to the pre-specified set of possibilities. Furthermore, most methods assume that the fibres are shifted, meaning a choice had to be made whether gaps or overlaps will be allowed during manufacturing [18].

Another approach that leads to manufacturable designs is to align the fibres in the direction of principal stress. This has been shown to reduce stress concentrations, and can also lead to reduced weight using the tailored fibre 
placement method [19, 20]. Using the load paths, or a hybrid combination of load paths and principal stress direction has also been used to design VSLs [21]. Continuous tow shearing is a new manufacturing method, leading to varying fibre angles without any gaps or overlaps, but with a thickness variation that is coupled with the change in fibre angle $[22,23]$. Using a genetic algorithm, coupled with a pattern-search algorithm, or using the infinite strip method large improvements in structural performance have been shown to be possible [24, 25]. A more comprehensive review of optimisation strategies can be found in Ghiasi et al. [26]

The lamination parameters are optimised in step one of the three-step optimisation approach, which has the disadvantage that a fibre angle retrieval step is needed. Enumeration has been used for constant stiffness laminates to match lamination parameters in terms of fibre angles [27]. If the number of layers gets too high, a layer-wise optimisation approach is used: first, the outer layer(s) are optimised, then the optimisation moves inward [28]. A genetic algorithm (GA) is sometimes used for variable stiffness laminates to retrieve fibre angles from the optimal lamination parameter distribution. Due to the computational cost of a GA, this is limited to a reasonably small number of variables $[29,30]$. Another approach is to try to match the in- and out-of-plane matrices as closely as possible using a combination of a GA and a modified Shepard's interpolation [31]. In other work, a real retrieval step was performed: the fibre angles were optimised using a combination of a $\mathrm{GA}$ and a gradient based optimiser to match the optimal stiffness distribution as closely as possible, while obeying a steering constraint [10].

In this paper, a method is discussed which combines fibre angle retrieval and fibre angle optimisation. First, the angles are retrieved based on the optimal stiffness distribution, next, starting from this fibre angle distribution, fibre angle optimisation is done. The paper is organised as follows: the optimisation approach is explained in section 2, after which the solution procedure is explained in section 3 . Then, two examples are worked out in more detail: a buckling optimisation example in section 4 and a stress optimisation in section 5 . The paper is concluded in section 6 .

\section{Optimisation approach}

In structural optimisation, the minimisation of an objective response (e.g., weight or compliance) subject to performance constraints (e.g., on stresses or displacements) is studied. More generally, the worst case response, for example in the case of multiple load cases, is optimised. Additional constraints not related to structural responses may also be imposed to guarantee certain properties of the design such as manufacturability. The following general problem formulation is considered:

$$
\begin{array}{cc}
\min _{x} & \max \left(f_{1}, f_{2}, \ldots, f_{n}\right) \\
\text { s.t. } & f_{n+1}, \ldots, f_{m} \leq 0 \\
& x_{i} \in \mathscr{D}_{i}
\end{array}
$$

The functions $f_{i}$ depend on the design variables; $f_{1}$ to $f_{n}$ denote structural responses that are optimised and $f_{n+1}$ to $f_{m}$ denote structural responses that are constrained. The feasible region is denoted by $\mathscr{D}$. This problem will be solved using successive approximations: one starts from a certain fibre angle distribution, constructs the approximations based on the optimal stiffness distribution, optimises the approximations and updates the approximations based on the new fibre angles. This is repeated until convergence is reached.

Structural responses, such as buckling loads, stiffness, and strength, are calculated using a finite element (FE) analysis. Since each FE analysis is computationally expensive, greater efficiency can be achieved by using structural approximations to reduce the required number of FE analyses [32,33]. The exact FE response $f$ is approximated in terms of the in- and out-of-plane stiffness matrices $A$ and $D$ and their reciprocals [1]:

$$
f^{(1)} \approx \sum_{n=1}^{N} \phi_{m}: A^{-1}+\phi_{b}: D^{-1}+\psi_{m}: A+\psi_{b}: D+c
$$

where the : operator represents the Frobenius inner product, $A: B=\operatorname{tr}\left(A \cdot B^{T}\right) ; \phi$ and $\psi$ are calculated from sensitivity analysis $[34,35]$. Subscripts $m$ and $b$ denote the membrane and bending parts respectively. This approximation is a generalisation of the linear-reciprocal approximations used in the convex linearisation method [36]. The approximations are convex functions in stiffness space provided that $\phi \geq 0$, a condition that is always satisfied by construction. The free term $c$ equals zero for many types of responses that enjoy homogeneity properties.

In the first step of the three-step approach, the approximations in (2) are parametrised in terms of the lamination parameters. The details of the lamination parameters optimisation are omitted here, they can be found in IJsselmuiden [1].

The fibre angles are the design variables for the second step of the three-step approach. Seen as a function of the fibre angles, the level one approximation, eq. (2), no longer has a simple mathematical form and is not 
generally convex, hence, a level two approximation is constructed based on the level one approximation in terms of the fibre angles:

$$
f^{(2)} \approx f_{0}^{(1)}+g \cdot \Delta \theta+\Delta \theta^{T} \cdot H \cdot \Delta \theta
$$

where $f_{0}^{(1)}$ denotes the value, $g$ the gradient and $H$ the Gauss-Newton part of the Hessian of the level one approximation at the approximation point.

While optimising this second level approximation, a steering constraint is also taken into account to make sure the found laminate is manufacturable. The steering is defined as

$$
\varsigma^{2}=\frac{2}{\Omega} \cdot \theta^{T} \cdot L \cdot \theta
$$

where $L$ is the standard FEM discretisation of the Laplacian. If this is taken to be the Laplacian of the complete layer, a global steering constraint is imposed, if the element Laplacian is used, a local steering constraint is imposed. The global steering constraint limits the number of gaps and overlaps, while the local steering makes sure the radius of curvature is not too small for the machine to lay down the fibre without wrinkling. The bound on the local steering $\varsigma^{U}$ is the inverse of the minimal steering radius needed; for example: a maximal steering value of $2 \mathrm{~m}^{-1}$, corresponds to a minimal steering radius of $500 \mathrm{~mm}$. The exact details on the fibre angle optimisation, including the steering constraints, can be found in Peeters et al. [33].

\section{Solution Procedure}

The sensitivities in the first iteration are calculated from this optimal stiffness distribution to incorporate the information about the optimum in terms of the stiffness matrices from step one of the three-step optimisation approach. This is the fibre angle retrieval step. The fibre angle optimisation does not stop after one iteration: the sensitivities are updated based on the current design to create a new level one approximation which is consequently optimised. The process is repeated until convergence, and is a full fibre angle optimisation. Convergence is defined in terms of the change in objective function. Unless specifically mentioned, a $1 \cdot 10^{-3}$ change in objective function is used as the convergence criterion. It is expected that the fibre angle optimisation will improve the structural performance: in regions where the stiffness distribution cannot be matched exactly, it is probably advantageous to find an optimal fibre angle distribution rather than approximating the optimal stiffness distribution.

The approximation used for fibre angle optimisation is guaranteed to be convex and separable, meaning every approximation has an optimum and the problem is not computationally expensive. To ensure convergence to a (local) optimum, every step needs to be an improvement (i.e., reduced objective and feasible constraints). One possibility to guarantee this is to make each approximation conservative, meaning it is strictly greater than the function it approximates at the new iterate. To achieve this, Svanberg proposed to add a positive function to the approximations, referred to as damping function in the remainder [37]. This damping function is scaled with a damping factor which is adjusted to make the approximations conservative. The damping factor is updated after each iteration: it is reduced if the new iterate is conservative with respect to the function it approximates, otherwise it is increased. The details of the damping function and damping factor can be found in Peeters et al. [33].

The solution procedure is shown in Figure 2, and is explained in algorithm 1.

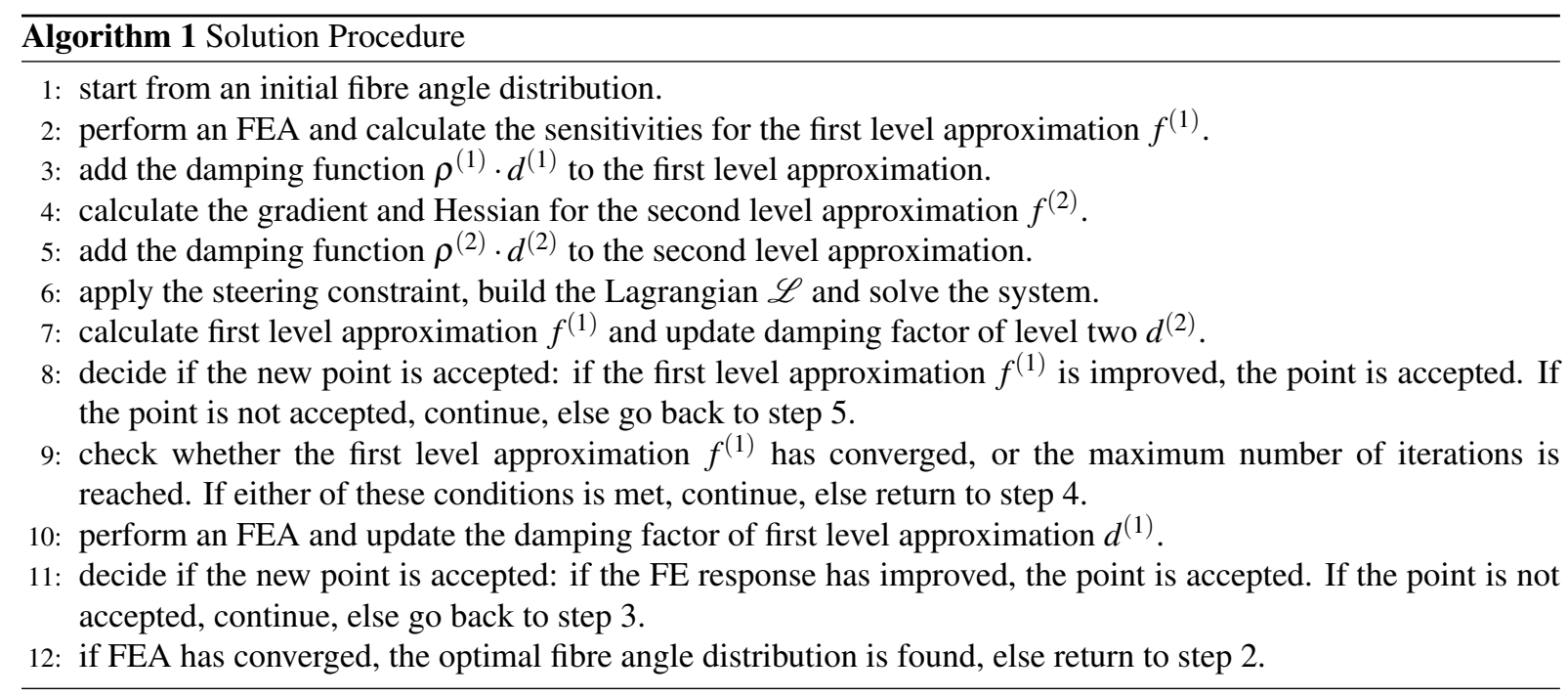




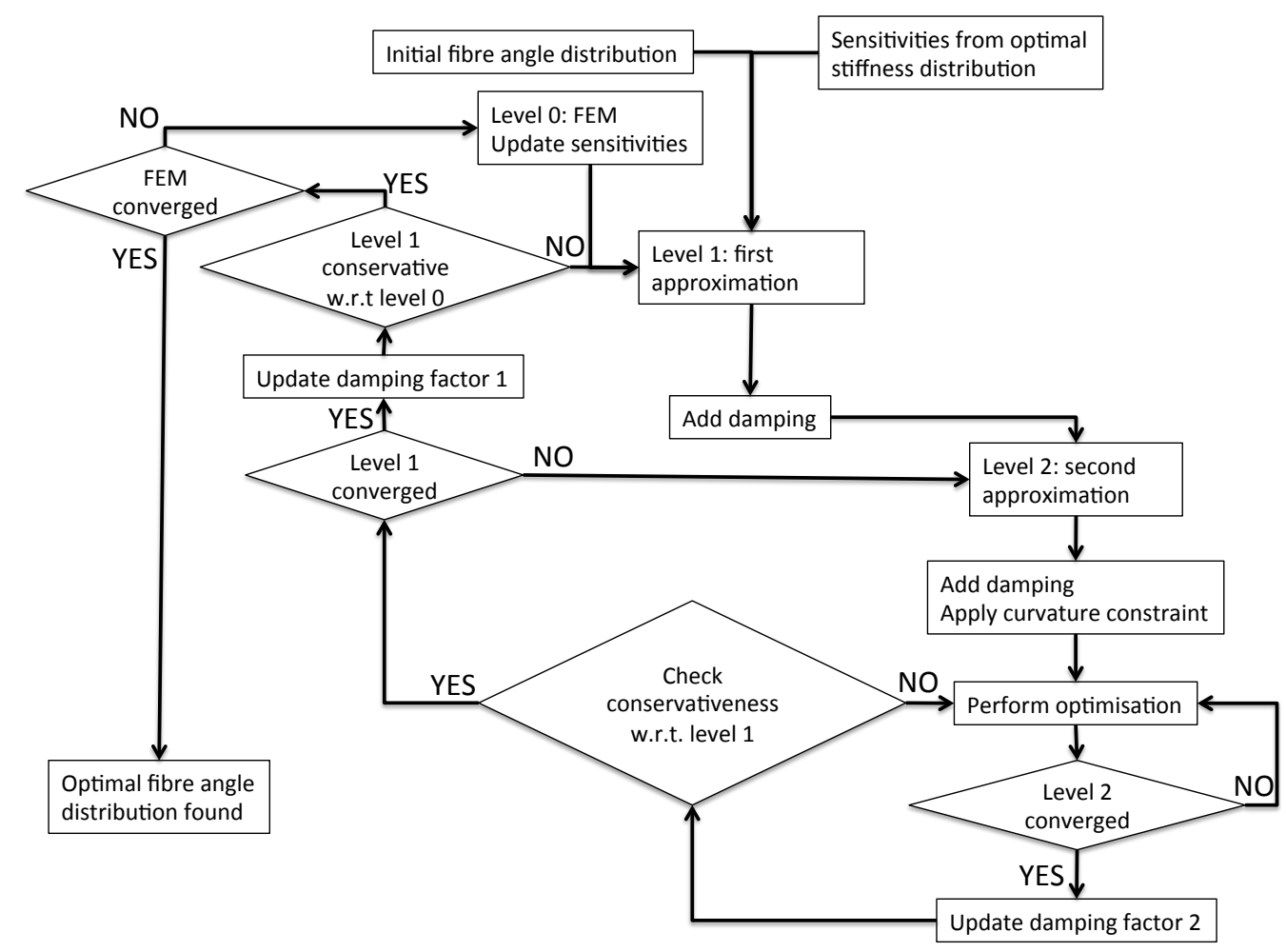

Figure 2. schematic overview of the code for a single optimisation step

\section{Buckling optimisation}

The first example is a singly-curved plate under compression. The buckling load of the plate is optimised with the constraint that the axial stiffness should be at least as much as that of a quasi-isotropic laminate of the same thickness. To take care of mode convergence and possible mode jumping, the lowest two buckling loads are simultaneously considered in the min-max formulation. The compliance constraint is set to avoid unstable post-buckling behaviour, which is dependent on the in-plane stiffness, while the buckling performance is mainly dependant on the out-of-plane stiffness. The plate is 600 by $400 \mathrm{~mm}$, with a sine-shaped height difference, which is maximal $75 \mathrm{~mm}$ in the middle. The left and right edge are constrained to remain straight, the left edge cannot move in $x$-direction, while the right edge is loaded with a unit compressive force. All edges are constrained to not move out-of-plane, and one node is extra constrained in $y$-direction to suppress rigid body modes. The material properties are as follows: $E_{1}=154 \mathrm{GPa}, E_{2}=10.8 \mathrm{GPa}, G_{12}=4.02 \mathrm{GPa}$ and $v_{12}=0.317$. The balanced layers are assumed to be next to each other, no thickness variation is taken into account. The physical laminate has 36 layers in total, but since symmetry and balance are imposed, only 9 design layers are part of the optimisation problem.

A mesh convergence study indicated that a sufficiently fine mesh is to use 24 elements in y-direction, and 36 in x-direction, leading to 1728 triangular elements and 925 nodes in the model used during optimisation. Due to the symmetry of the problem, only a quarter of the plate will be used during the optimisation, reducing the number of nodes in the optimisation to 247 , and the number of elements to 432 . The complete model is used for the FE analysis and sensitivity calculation.

When performing the optimisation in stiffness space, the optimal buckling loads found are 2.2432 and 2.4656 times the lowest QI buckling load while the stiffness is 1.0001 times the QI stiffness.

\subsection{Influence of initial fibre angle retrieval}

The optimisation will be performed twice to assess the computational advantages of the fibre angle retrieval step. The first time, the initial approximations are calculated at a user-specified initial fibre angle distribution. The second time, the initial approximations are calculated at the optimal stiffness distribution produced by lamination parameters optimisation. When starting from the sensitivities of the optimal stiffness distribution, the first iteration is a fibre angle retrieval step. Five level one iterations are allowed, following previous work done by the authors [33]. Only a local steering constraint of $3 \mathrm{~m}^{-1}$, corresponding to a minimal turning radius of $333 \mathrm{~mm}$, is used for this investigation. In the following, the buckling load and stiffness values are normalised with respect to the corresponding values of a quasi-isotropic laminate of the same thickness. 
Table 1. Overview of the results using different initial sensitivities

\begin{tabular}{|c||c|c||c|c||c|c|}
\hline $\begin{array}{c}\text { initial } \\
\text { fibre } \\
\text { angles [deg] }\end{array}$ & $\begin{array}{c}\text { optimal } \\
\text { buckling load } \\
\text { without fibre } \\
\text { angle retrieval [-] }\end{array}$ & $\begin{array}{c}\text { number of } \\
\text { FEAs without } \\
\text { fibre angle } \\
\text { retrieval }\end{array}$ & $\begin{array}{c}\text { optimal } \\
\text { buckling load } \\
\text { with fibre } \\
\text { angle retrieval [-] }\end{array}$ & $\begin{array}{c}\text { number of } \\
\text { FEAs with } \\
\text { fibre angle } \\
\text { retrieval }\end{array}$ & $\begin{array}{c}\text { difference } \\
\text { in } \\
\text { buckling load }\end{array}$ & $\begin{array}{c}\text { difference } \\
\text { FEAs } \\
\text { used }\end{array}$ \\
\hline 0.1 & 1.7574 & 14 & 1.7696 & 8 & $+0.7 \%$ & -6 \\
1 & 1.7620 & 21 & 1.7671 & 5 & $+0.3 \%$ & -16 \\
10 & 1.7608 & 13 & 1.7486 & 8 & $-0.7 \%$ & -5 \\
20 & 1.7628 & 11 & 1.7645 & 5 & $+0.1 \%$ & -6 \\
\hline
\end{tabular}

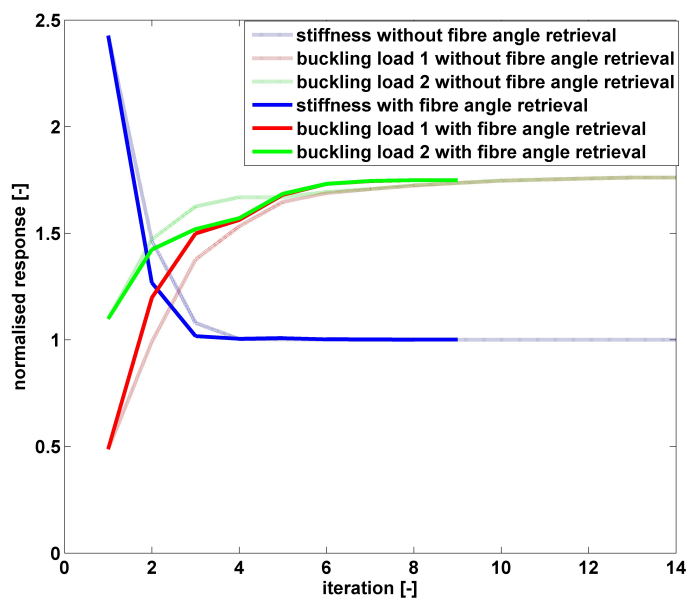

Figure 3. convergence history with and without initial fibre angle retrieval step

The results are shown in Table 1. The initial fibre angle distribution is assumed to be uniform for all layers and nodes. The initial angle is shown in the first column. The optimal buckling load, and number of FEAs used when no retrieval step is applied are shown in the second and third columns. The optimal buckling load, and number of FEAs used with fibre angle retrieval activated are shown in the fourth and fifth columns. The difference in optimal buckling load and FEAs used between the two optimisation strategies are shown in the last two columns. The stiffness is not shown in Table 1 to save space, but it was always equal to the QI stiffness, or slightly higher, also only the critical buckling load is shown; the second buckling load was always close to it but not identical. It can be seen from Table 1 that the influence of the initial fibre angles is limited: a maximum change of $1.2 \%$ is observed. The solution procedure seems to be robust to initial conditions. When using fibre angle retrieval, the number of FEAs is always decreased, in most cases it is halved. The difference in optimal buckling load due to the different initial sensitivities is negligible, less then $1 \%$, and may be explained by the stopping criterion in a gradient-based algorithm.

Observing the convergence history, shown in Figure 3, the largest improvement is seen to be made in the first level one step. When fibre angle retrieval is used, less iterations are needed to 'fine-tune' the design. This could be because in the firs step the stiffness distribution is already getting to the best one based on the optimal stiffness, while when starting from the initial fibre angle distribution, there is more fine-tuning to be done.

It is interesting to inspect the convergence of the level two optimisation. To check this, an FEA is done after each level two update until convergence is reached. This is done for an initial fibre angle of 1,10 and $20^{\circ}$. For some cases convergence is established fairly quickly and the 5 inner iteration limit imposed earlier is adequate. For the case of a $10^{\circ}$ initial angle the convergence is slower and it is possible that more inner iterations are beneficial. The reason the convergence is not monotonous (i.e., constantly decreasing response) is that during level two updates, level one approximations are monitored and are guaranteed to decrease. It can happen that the level two updates wander out of the range of validity of level one approximations leading to non-monotone convergence of the FEA results.

A maximum improvement over QI of $60 \%$ is found when fibre angle retrieval is used on its own. After updating the approximations and converging the full fibre angle optimisation, a $76 \%$ improvement is found. Thus, the best 


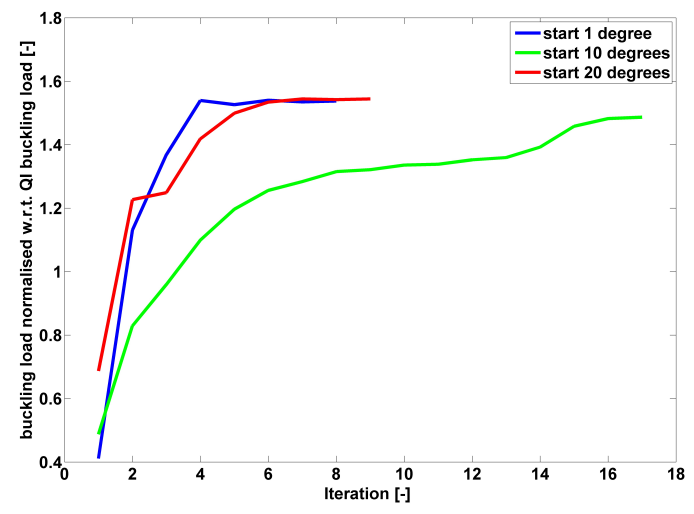

Figure 4. FE response after each level 2 optimisation (during fibre angle retrieval)

Table 2. Overview of the results using different local steering constraints

\begin{tabular}{|c|c|c|c|c|c|c|c|}
\hline $\begin{array}{c}\text { maximum } \\
\text { local } \\
\text { steering } \\
{\left[\mathrm{m}^{-1}\right]}\end{array}$ & $\begin{array}{c}\text { optimal } \\
\text { buckling } \\
\text { load after } \\
\text { fibre angle } \\
\text { retrieval [-] }\end{array}$ & $\begin{array}{c}\text { optimal } \\
\text { buckling } \\
\text { load 1 } \\
{[-]}\end{array}$ & $\begin{array}{c}\text { optimal } \\
\text { buckling } \\
\text { load 2 } \\
{[-]}\end{array}$ & $\begin{array}{c}\text { optimal } \\
\text { stiffness } \\
{[-]}\end{array}$ & $\begin{array}{c}\text { number } \\
\text { of FEAs }\end{array}$ & $\begin{array}{c}\text { difference } \\
\text { w.r.t. } \\
\text { optimum } \\
\text { after fibre angle } \\
\text { retrieval }\end{array}$ & $\begin{array}{c}\text { difference } \\
\text { w.r.t. optimal } \\
\text { stiffness } \\
\text { distribution }\end{array}$ \\
\hline 1 & 1.1210 & 1.3731 & 1.3735 & 1.0012 & 4 & $+22.5 \%$ & $-38.8 \%$ \\
2 & 1.2894 & 1.5749 & 1.5752 & 1.0005 & 5 & $+22.1 \%$ & $-29.8 \%$ \\
3 & 1.4867 & 1.7474 & 1.7474 & 1.0008 & 5 & $+17.5 \%$ & $-22.1 \%$ \\
4 & 1.6024 & 1.8846 & 1.8852 & 1.0010 & 4 & $+17.6 \%$ & $-16.0 \%$ \\
5 & 1.7550 & 1.9830 & 1.9835 & 1.0007 & 5 & $+13.0 \%$ & $-11.6 \%$ \\
\hline
\end{tabular}

optimisation strategy seems to be to combine the fibre angle retrieval and angle optimisation. This way a better performance is achieved while keeping the cost, in terms of number of FEAs, low.

\subsection{Influence of manufacturing constraints}

The influence of manufacturing constraints on how close the optimal stiffness response can be approached is investigated. First, the optimisation is performed for different levels of the maximum local steering, there is no constraint on the global steering. The results, for a local steering of $1 \mathrm{~m}^{-1}$ up to $5 \mathrm{~m}^{-1}$, are shown in Table 2, where the maximum local steering is shown in the first column, the optimal buckling load with only fibre angle retrieval is shown in the second column, the optimal buckling loads and stiffness are shown in columns three to five. The sixth column lists the number of FEAs needed, the seventh column indicates the improvement obtained by full angle optimisation over only angle retrieval, and the last column lists the difference in performance with respect to the stiffness optimum. Note that the level two optimisation in the fibre angle retrieval step was allowed to converge fully to ensure a fair comparison between the results with and without full fibre angle optimisation.

The higher the allowed steering, the higher is the improvement in buckling load. Furthermore, it seems that the difference obtained by performing fibre angle optimisation after the retrieval step diminishes. This is due to the better fit that can be obtained at higher steering values.

The Pareto front trading off manufacturability versus structural performance is shown in Figure 5, here, the minimum turning radius represents manufacturability and the buckling load represents performance. The Pareto front is not convex which is typical of the highly non-convex buckling optimisation problem. It seems that initially large performance improvements can be made by decreasing the minimum steering radius of curvature; the improvements becoming gradually less siginficant for tighter radii. The response does seem to converge to the stiffness optimum as expected.

As discussed earlier, the local steering constraint is imposed to guarantee a minimum turning radius. This is not the only manufacturing consideration; gaps/overlaps due to fibre divergence/convergence may develop. These are considered manufacturing defects and it is desirable to minimise them. Detailed prediction of gaps and overlaps requires full simulation of the fibre-placement process. Embedding a full simulation in the optimisation process would not be feasible especially since additional geometric freedom, not related to the fibre angle distribution, is 


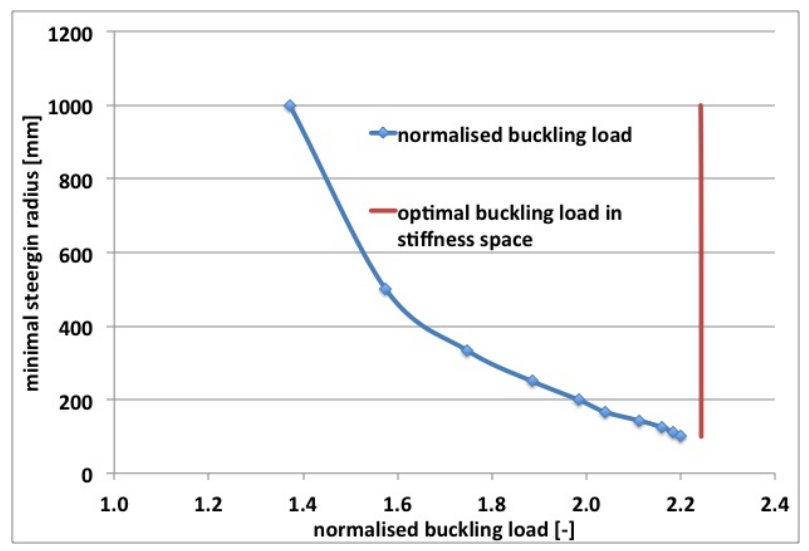

Figure 5. normalised buckling load versus the minimal steering radius

Table 3. Overview of the results using different global steering constraints

\begin{tabular}{|c|c|c|c|c|c|c|c|}
\hline $\begin{array}{c}\text { maximum } \\
\text { global } \\
\text { steering } \\
{\left[\mathrm{m}^{-1}\right]}\end{array}$ & $\begin{array}{c}\text { optimal } \\
\text { buckling } \\
\text { load after } \\
\text { fibre angle } \\
\text { retrieval [-] }\end{array}$ & $\begin{array}{c}\text { optimal } \\
\text { buckling } \\
\text { load 1 } \\
{[-]}\end{array}$ & $\begin{array}{c}\text { optimal } \\
\text { buckling } \\
\text { load 2 } \\
{[-]}\end{array}$ & $\begin{array}{c}\text { optimal } \\
\text { stiffness } \\
{[-]}\end{array}$ & $\begin{array}{c}\text { number } \\
\text { of FEAs }\end{array}$ & $\begin{array}{c}\text { difference } \\
\text { w.r.t. } \\
\text { optimum } \\
\text { after fibre angle } \\
\text { retrieval }\end{array}$ & $\begin{array}{c}\text { difference } \\
\text { w.r.t. optimal } \\
\text { stiffness } \\
\text { distribution }\end{array}$ \\
\hline 0.01 & 1.0307 & 1.2132 & 1.2132 & 1.1067 & 6 & $+17.7 \%$ & $-45.9 \%$ \\
0.5 & 1.0519 & 1.2888 & 1.2888 & 1.0011 & 5 & $+22.5 \%$ & $-42.6 \%$ \\
1 & 1.1274 & 1.3992 & 1.3999 & 1.0011 & 4 & $+24.1 \%$ & $-37.6 \%$ \\
1.5 & 1.2292 & 1.5192 & 1.5196 & 1.0007 & 5 & $+23.6 \%$ & $-32.3 \%$ \\
2 & 1.3532 & 1.6330 & 1.6333 & 1.0007 & 5 & $+20.7 \%$ & $-27.2 \%$ \\
2.5 & 1.4819 & 1.7436 & 1.7441 & 1.0006 & 6 & $+17.7 \%$ & $-22.3 \%$ \\
3 & 1.5724 & 1.8034 & 1.8046 & 1.0005 & 4 & $+14.7 \%$ & $-19.6 \%$ \\
3.5 & 1.0181 & 1.8467 & 1.8481 & 1.0013 & 8 & $+81.4 \%$ & $-17.7 \%$ \\
4 & 1.6024 & 1.8846 & 1.8852 & 1.0010 & 4 & $+17.6 \%$ & $-16.0 \%$ \\
\hline
\end{tabular}

available to the designer to space the fibres in a way that best avoids gaps and overlaps. We propose to use the global steering as a surrogate measure of the extent of local defects in the laminate. A reasonable value for the local steering, $4 \mathrm{~m}^{-1}$ corresponding to a minimal turning radius of $250 \mathrm{~mm}$, is selected and fixed. The upper bound on global steering is varied to investigate the effect on optimal performance.

The results are shown in Table 3, where the global steering constraint is shown in the first column, the optimal buckling load after fibre angle retrieval is shown in the second column. The optimal buckling loads and stiffness are shown in columns three to five. The number of FEAs is shown in the sixth column, the difference obtained by full angle optimisation is shown in the seventh column while the last column indicates the difference with respect to the optimal stiffness design. The lowest value used for global steering is small enough to be considered as the optimal straight-fibre, constant stiffness, laminate. Up to 55\% improvement in the buckling load can be achieved by manufacturable steered laminates. The difference between fibre angle retrieval and full optimisation is mostly around $20 \%$ supporting our earlier observations.

\section{Strength optimisation}

The second example problem is the taken from Khani et al. [38]. A plate with a circular cut-out loaded in tension is optimised for strength. The plate is 400 by $400 \mathrm{~mm}$, with a large circular cut-out with a diameter of $200 \mathrm{~mm}$ at the centre. The plate is simply supported all around, with all edges constrained to remain straight. After taking the symmetry into account, the plate was discretised into 217 triangular elements with 132 nodes. The material stiffness properties are as follows: $E_{1}=142.9 \mathrm{GPa}, E_{2}=10.3 \mathrm{GPa}, G_{12}=7.2 \mathrm{GPa}$ and $v_{12}=0.27$. The failure is defined using the conservative omni-strain envelope [39, 38,40]. The total laminate has a thickness of $4.6 \mathrm{~mm}$, meaning there are 24 layers. As in the previous example, the laminate is assumed the be balanced and symmetric, leading to 6 design layers. 
Table 4. Overview of the results using different local steering constraints using $1 \cdot 10^{-3}$ as stopping criterion

\begin{tabular}{|c|c|c|c|c|c|}
\hline $\begin{array}{c}\text { maximum } \\
\text { local } \\
\text { steering } \\
{\left[\mathrm{m}^{-1}\right]}\end{array}$ & $\begin{array}{c}\text { minimum } \\
\text { factor of } \\
\text { safety after } \\
\text { fibre angle } \\
\text { retrieval [-] }\end{array}$ & $\begin{array}{c}\text { optimal } \\
\text { factor of } \\
\text { safety } \\
{[-]}\end{array}$ & $\begin{array}{c}\text { number } \\
\text { of FEAs }\end{array}$ & $\begin{array}{c}\text { difference } \\
\text { w.r.t. } \\
\text { optimum } \\
\text { after fibre angle } \\
\text { retrieval }\end{array}$ & $\begin{array}{c}\text { difference } \\
\text { w.r.t. optimal } \\
\text { stiffness } \\
\text { distribution }\end{array}$ \\
\hline 0.01 & 1.288 & 1.578 & 4 & $+22.5 \%$ & $-18.8 \%$ \\
1 & 1.345 & 1.811 & 10 & $+34.6 \%$ & $-6.8 \%$ \\
2 & 1.357 & 1.830 & 12 & $+34.9 \%$ & $-5.9 \%$ \\
3 & 1.395 & 1.869 & 15 & $+34.0 \%$ & $-3.9 \%$ \\
\hline
\end{tabular}

Table 5. Overview of the results using different local steering constraints using $3 \cdot 10^{-3}$ as stopping criterion

\begin{tabular}{|c|c|c|c|c|c|c|}
\hline $\begin{array}{c}\text { maximum } \\
\text { local } \\
\text { steering } \\
{\left[\mathrm{m}^{-1}\right]}\end{array}$ & $\begin{array}{c}\text { minimum } \\
\text { factor of } \\
\text { safety after } \\
1 \text { step [-] }\end{array}$ & $\begin{array}{c}\text { optimal } \\
\text { factor of } \\
\text { safety } \\
{[-]}\end{array}$ & $\begin{array}{c}\text { number } \\
\text { of FEAs }\end{array}$ & $\begin{array}{c}\text { difference } \\
\text { w.r.t. } \\
\text { optimum } \\
\text { after 1 step }\end{array}$ & $\begin{array}{c}\text { difference } \\
\text { w.r.t. optimal } \\
\text { stiffness } \\
\text { distribution }\end{array}$ & $\begin{array}{c}\text { difference } \\
\text { w.r.t. tighter } \\
\text { convergence } \\
\text { criterion }\end{array}$ \\
\hline 1 & 1.345 & 1.688 & 5 & $+25.5 \%$ & $-13.2 \%$ & $-6.8 \%$ \\
2 & 1.357 & 1.709 & 5 & $+25.9 \%$ & $-12.1 \%$ & $-6.6 \%$ \\
3 & 1.395 & 1.746 & 5 & $+25.2 \%$ & $-10.2 \%$ & $-6.6 \%$ \\
\hline
\end{tabular}

The load is chosen such that the QI design has a minimum factor of safety of 1 . When performing the optimisation in terms of the lamination parameters, the optimal design has a minimum factor of safety of 1.944. Only the effect of the local steering constraint will be investigated. The optimisation will be performed for straight fibres, by setting the maximum local steering to $0.01 \mathrm{~m}^{-1}$, and for local steering bounds from 1 to $3 \mathrm{~m}^{-1}$. The results are shown in Table 4, where the local steering constraint is shown in the first column. The maximum failure index after angle retrieval and at the full optimisation optimum are shown in the second and third column, the fourth column gives the number of FEAs needed to get to the optimum, the difference from angle retrieval due to full optimisation is shown in column six, the last column indicates the difference with respect to the optimum stiffness distribution.

The small number of FEAs needed for the straight-fibre laminate stands out: only 4. Although the approximations are only updated four times, there is a clear advantage in updating them. The large increase in safety factor after the fibre angle retrieval is due to the bad matching in the vicinity of the hole: updating the approximations makes sure the optimal fibre angle is found, as opposed to a suboptimal lamination parameter match. The number of FEAs is low due to the limited design space: no steering is allowed, thus not much 'fine-tuning' can be done.

When steering is allowed, the increase in safety factor is more significant. This comes at the cost of significantly more FEAs. Since steering is allowed, a wider design space is available to the optimiser, and a lot of 'fine-tuning' can be done. If the convergence criterion is chosen to be $3 \cdot 10^{-3}$ instead of $1 \cdot 10^{-3}$, fewer FEAs will be needed, but at the expense of reduced optimal performance. The results are shown in Table 5. This table has the same layout as Table 4 with one column added where the difference with respect to the tighter convergence criterion is given.

What really stands out is that even with a relatively limited steering of $3 m^{-1}$, one can already get within $4 \%$ of the optimal stiffness distribution. This can be explained by the relatively low load redistribution that has to be done for the stress optimisation. For buckling optimisation, all the load is redistributed to the sides, while for the strength, the load is gently nudged away from the cut-out, possibly because of the geometry: the cut-out is large compared to the total size of the plate.

To see what is happening in terms of the stiffness distribution, the $V_{1}$ and $V_{3}$ distribution are shown in Figure 6. The optimal stiffness distribution found is shown at the top, the lamination parameters after the fibre angle retrieval step are shown in the middle, and the optimal fibre angle distribution is shown in the bottom. A local steering constraint of $3 m^{-1}$ is used for the fibre angle retrieval and optimisation. In the top figures, it can be seen there is a lot of difference in stiffness from one point to the next, which causes the stiffness distribution after the fibre angle retrieval step to be very far away from the optimal, not knowing what places should be matched more accurately. When looking at the stiffness distribution for the fibre angle optimum, it can be seen that the stiffness change is smoother, and the sides and middle are matched exactly, while the part in between looks completely 
different. Although the difference in stiffness distribution is very large, the factor of safety only differs by $3 \%$ : the stiffness optimisation allows very large changes in stiffness from one point to the next for a small increase in performance, while this is constrained in the fibre angle optimisation.

\section{Conclusion}

A method to optimise the fibre angle distributions of variable stiffness laminates is proposed. The proposed method integrates a fibre angle retrieval step with the fibre angle optimisation procedure. The fibre angles are retrieved using the approximation based on the optimal stiffness distribution. Due to a steering constraint, which limits the rate of change in fibre angles, the stiffness distribution cannot, in general, be matched exactly. Hence, fibre angle optimisation is performed, using successive approximations, after the fibre angle retrieval step, until convergence is reached. Two examples are used to demonstrate the approach. The first example shown is a single curved plate under compression, optimised for buckling with a stiffness constraint. The second example is a square plate with a circular hole in the centre under tension, optimised for maximum strength. The results indicate a strong trade-off between manufacturability, measured by the bound on steering, and performance.

The results show that by incorporating a fibre-angle retrieval step, the number of FEAs goes down significantly, usually it is halved, compared to starting from a user-specified initial stacking sequence. While the fibre angle retrieval leads to improved numerical efficiency, the results indicate that fibre angle retrieval in itself is not sufficient to obtain the best performance. Further full fibre angle optimisation steps, in the buckling example, led on average to $15-20 \%$ improvement in performance over fibre angle retrieval. The strength optimisation example showed that even larger improvements are possible by performing full fibre angle optimisation, almost $35 \%$ on average. The best strategy seems to be to combine fibre angle retrieval and angle optimisation. This way a better performance is achieved while limiting the number of FE analyses.

Overall, integration of fibre-angle retrieval in the full fibre angle optimisation process reduces the computational cost. This gain in efficiency will allow designers to study the trade-off between manufacturability and performance carefully and to select the most promising design(s) depending on the relative importance of both considerations.

\section{Acknowledgements}

This work is supported by the CANAL (CreAting Non-conventionAl Laminates) Project, part of the European Union Seventh Framework Program.

\section{References}

[1] IJsselmuiden, S. T., Optimal design of variable stffness composite structures using lamination parameters, Ph.D. thesis, Delft University of Technology, 2011.

[2] Peeters, D., van Baalen, D., and Abdalla, M., "Combining topology and lamination parameter optimisation," Structural and Multidisciplinary Optimization, 2015, pp. 1-16.

[3] Autio, M., "Determining the real lay-up of a laminate corresponding to optimal lamination parameters by genetic search," Structural and Multidisciplinary Optimization, Vol. 20, No. 4, 2000, pp. 301-310.

[4] Setoodeh, S., Blom, A., Abdalla, M., and Gürdal, Z., "Generating Curvilinear Fiber Paths from Lamination Parameters Distribution," 47th AIAA/ASME/ASCE/AHS/ASC Structures, Structural Dynamics, and Materials Conference, American Institute of Aeronautics and Astronautics, 2014/12/01 2006.

[5] van Campen, J. and Gürdal, Z., "Retrieving Variable Stiffness Laminates from Lamination Parameters Distribution," 50th AIAA/ASME/ASCE/AHS/ASC Structures, Structural Dynamics, and Materials Conference, American Institute of Aeronautics and Astronautics, 2014/12/01 2009.

[6] Blom, A. W., Abdalla, M. M., and Gürdal, Z., "Optimization of course locations in fiber-placed panels for general fiber angle distributions," Composites Science and Technology, Vol. 70, No. 4, 2010, pp. $564-570$.

[7] Lopes, C., Gürdal, Z., and Camanho, P., "Tailoring for strength of composite steered-fibre panels with cutouts," Composites Part A: Applied Science and Manufacturing, Vol. 41, No. 12, 2010, pp. 1760 - 1767.

[8] Gürdal, Z., Tatting, B., and Wu, C., "Variable stiffness composite panels: Effects of stiffness variation on the in-plane and buckling response," Composites Part A: Applied Science and Manufacturing, Vol. 39, No. 5, 2008, pp. $911-922$. 


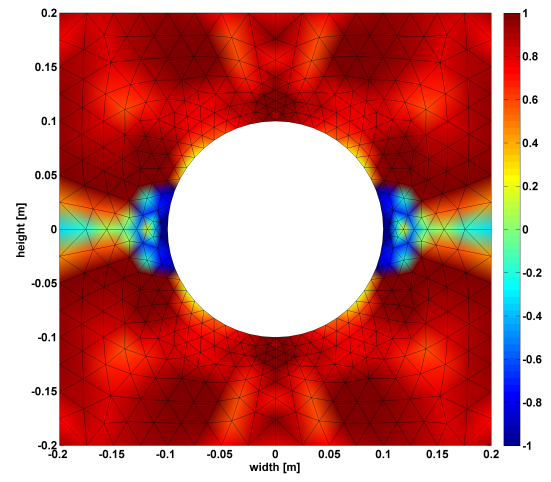

(a) $V_{1}$ distribution for optimal stiffness

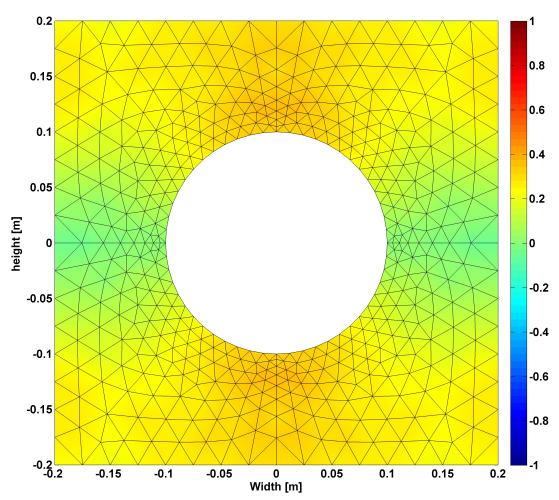

(c) $V_{1}$ distribution after fibre angle retrieval step for maximum local steering of $3 m^{-1}$

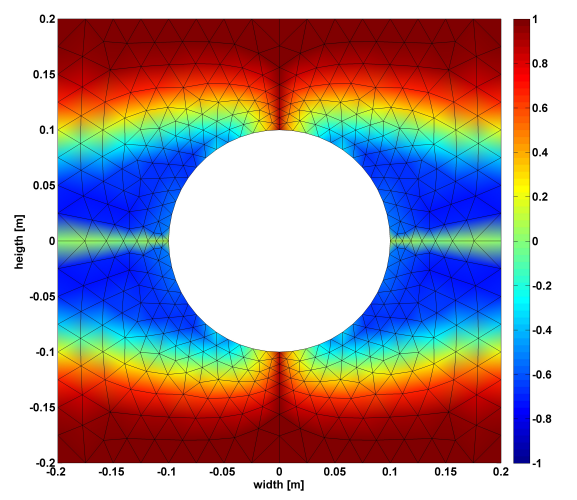

(e) $V_{1}$ distribution after fibre angle optimisation for maximum local steering of $3 m^{-1}$

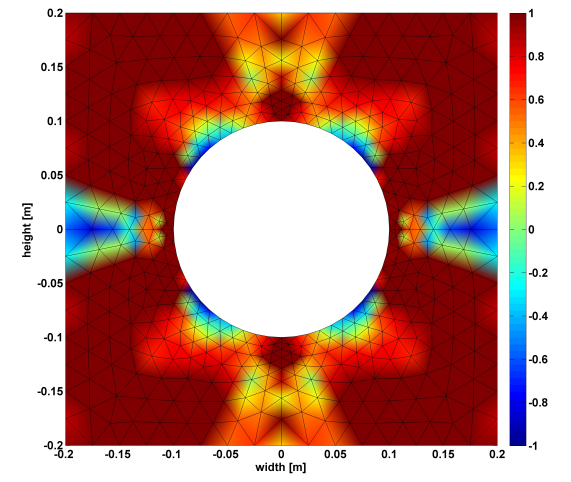

(b) $V_{3}$ distribution for optimal stiffness

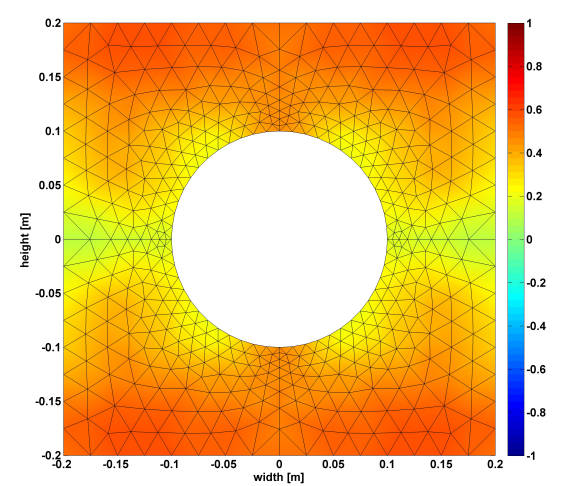

(d) $V_{3}$ distribution after fibre angle retrieval step for maximum local steering of $3 m^{-1}$

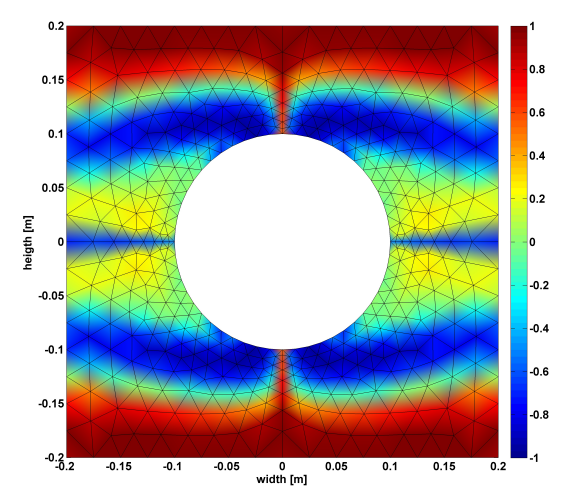

(f) $V_{3}$ distribution after fibre angle optimisation for maximum local steering of $3 m^{-1}$

Figure 6. in-plane lamination parameters for different cases 
[9] Hyer, M. W. and Charette, R. F., "Use of curvilinear fiber format in composite structure design," AIAA Journal, Vol. 29, No. 6, 2014/12/01 1991, pp. 1011-1015.

[10] van Campen, J. M., Kassapoglou, C., and Gürdal, Z., "Generating realistic laminate fiber angle distributions for optimal variable stiffness laminates," Composites Part B: Engineering, Vol. 43, No. 2, 2012, pp. 354 360.

[11] Stodieck, O., Cooper, J., Weaver, P., and Kealy, P., "Improved aeroelastic tailoring using tow-steered composites," Composite Structures, Vol. 106, 2013, pp. 703-715, cited By 7.

[12] Ungwattanapanit, T. and Baier, H., "Postbuckling analysis and optimization of stiffened fuselage panels utilizing variable-stiffness laminates," Congress of the International Council of the Aeronautical Sciences, ICAS [29., 2014, St. Petersburg], 2014.

[13] Liu, W. and Butler, R., "Buckling optimization for composite panels with elastic tailoring," 49th AIAA/ASME/ASCE/AHS/ASC Structures, Structural Dynamics and Materials Conference, April 2008.

[14] Coburn, B., Wu, Z., and Weaver, P., "Buckling analysis of stiffened variable angle tow panels," Composite structures, Vol. 111, No. 1, 2014, pp. 259-270.

[15] Nagendra, S., Kodiyalam, S., Davis, J. E., and Parthasarathy, V. N., "Optimization of Tow Fiber Paths for Composite Design," 36th AIAA/American Society of Mechanical Engineers/American Society of Civil Engineers/American Helicopter Society/ Society for Composites Structures, Structural Dynamics, and Materials Conference, 1995.

[16] van den Brink WM, Vankan, W., and Maas, R., "Buckling optimized variable stiffness laminates for a composite fuselage window section," 28th international congress of the aeronautical sciences, 2012.

[17] Wu, Z., Weaver, P. M., Raju, G., and Kim, B. C., "Buckling analysis and optimisation of variable angle tow composite plates," Thin-Walled Structures, Vol. 60, No. 0, 2012, pp. 163 - 172.

[18] Gürdal, Z., Tatting, B., and Wu, K., "Tow-Placement Technology and Fabrication Issues for Laminated Composite Structures," 46th AIAA/ASME/ASCE/AHS/ASC Structures, Structural Dynamics and Materials Conference, American Institute of Aeronautics and Astronautics, 2014/11/30 2005.

[19] Crothers, P., Drechsler, K., Feltin, D., Herszberg, I., and Kruckenberg, T., "Tailored fibre placement to minimise stress concentrations," Composites Part A: Applied Science and Manufacturing, Vol. 28, No. 7, 1997, pp. $619-625$.

[20] Richter, E., Uhlig, K., Spickenheuer, A., Bittrich, L., Maäder, E., and Heinrich, G., "thermoplastic composite parts based on online spun commingled hybrid yarns with continuous curvilinear fibre patterns," 16th European Conference on Composite Materials, June 2014.

[21] Tosh, M. and Kelly, D., "On the design, manufacture and testing of trajectorial fibre steering for carbon fibre composite laminates," Composites Part A: Applied Science and Manufacturing, Vol. 31, No. 10, 2000, pp. $1047-1060$.

[22] Kim, B., Potter, K., and Weaver, P., Multi-tow shearing mechanism for high-speed manufacturing of variable angle tow composites, 2012, Venice, IT.

[23] Kim, B., Potter, K., and Weaver, P., "Continuous tow shearing for manufacturing variable angle tow composites,” Composites, Part A: Applied Science and Manufacturing, Vol. 43, No. 8, 2012, pp. 1347-1356.

[24] Groh, R. and Weaver, P., "Mass Optimization of Variable Angle Tow, Variable Thickness Panels with Static Failure and Buckling Constraints," 56th AIAA/ASME/ASCE/AHS/ASC Structures, Structural Dynamics and Materials Conference, January 2015.

[25] Liu, W. and Butler, R., "Buckling Optimization of Variable-Angle-Tow Panels Using the Infinite-Strip Method," AIAA Journal, Vol. 51, No. 6, 2015/01/29 2013, pp. 1442-1449.

[26] Ghiasi, H., Fayazbakhsh, K., Pasini, D., and Lessard, L., "Optimum stacking sequence design of composite materials Part II: Variable stiffness design,” Composite Structures, Vol. 93, No. 1, 2010, pp. 1 - 13. 
[27] Honda, S., Narita, Y., and Sasaki, K., "Discrete Optimization for Vibration Design of Composite Plates by Using Lamination Parameters," Advanced Composite Materials, Vol. 18, No. 4, 2009, pp. 297-314.

[28] Narita, Y., "Layerwise optimization for the maximum fundamental frequency of laminated composite plates," Journal of Sound and Vibration, Vol. 263, No. 5, 2003, pp. 1005 - 1016, Arthur W. Leissa 70th Birthday Issue.

[29] Liu, S., Hou, Y., Sun, X., and Zhang, Y., “A two-step optimization scheme for maximum stiffness design of laminated plates based on lamination parameters," Composite Structures, Vol. 94, No. 12, 2012, pp. 3529 3537.

[30] Raju, G., White, S., Wu, Z., and Weaver, P., Optimal Postbuckling Design of Variable Angle Tow Composites using Lamination Parameters, American Institute of Aeronautics and Astronautics, 2015/03/30 2015.

[31] "Stacking Sequence Optimization of Blended Composites using a Modified Shepard's Method and Stacking Sequence Tables," composite structures, submitted.

[32] de Wit, A. and van Keulen, F., "Numerical Comparison of Multi-Level Optimization Techniques," 48th AIAA/ASME/ASCE/AHS/ASC Structures, Structural Dynamics, and Materials Conference, American Institute of Aeronautics and Astronautics, 2014/11/11 2007.

[33] Peeters, D., Hesse, S., and Abdalla, M., "Stacking Sequence Optimisation of Variable Stiffness Laminates with Manufacturing Constraints," Composites Part B: Engineering, submitted.

[34] Haftka, R. and Gürdal, Z., Elements of Structural Optimization, Contributions to Phenomenology, Springer Netherlands, 1992.

[35] Kumar, V., Lee, S.-J., and German, M., "Finite element design sensitivity analysis and its integration with numerical optimization techniques for structural design," Computers and Structures, Vol. 32, No. 34, 1989, pp. $883-897$.

[36] Fleury, C., "CONLIN: An efficient dual optimizer based on convex approximation concepts," Structural optimization, Vol. 1, No. 2, 1989, pp. 81-89.

[37] Svanberg, K., "a class of globally convergent optimization methods based on conservative convex separable approximations," Siam J. optim, Vol. 2, 2002, pp. 555-573.

[38] Khani, A., IJsselmuiden, S., Abdalla, M., and Grdal, Z., "Design of variable stiffness panels for maximum strength using lamination parameters," Composites Part B: Engineering, Vol. 42, No. 3, 2011, pp. 546 - 552.

[39] Ijsselmuiden, S. T., Abdalla, M. M., and Gürdal, Z., "Implementation of Strength-Based Failure Criteria in the Lamination Parameter Design Space,” AIAA Journal, Vol. 46, No. 7, 2015/04/15 2008, pp. 1826-1834.

[40] Tsai, S. W. and Melo, J. D. D., “An invariant-based theory of composites," Composites Science and Technology, Vol. 100, No. 0, 2014, pp. $237-243$. 